\title{
The twisted Grassmann graph is the block graph of a design
}

\author{
Akihiro Munemasa Vladimir D. Tonchev
}

\begin{abstract}
In this note, we show that the twisted Grassmann graph constructed by Van Dam and Koolen is the block graph of the design constructed by Jungnickel and Tonchev. We also show that the full automorphism group of the design is isomorphic to that of the twisted Grassmann graph.
\end{abstract}

Keywords : distance-regular graph, Grassmann graph, projective geometry, design

\section{Introduction}

Let $V$ be a $(2 e+1)$-dimensional vector space over $\operatorname{GF}(q)$. If $W$ is a subset of $V$ closed under multiplication by the elements of $\operatorname{GF}(q)$, then we denote by $[W]$ the set of 1-dimensional subspaces (projective points) contained in $W$. We also denote by $\left[\begin{array}{c}W \\ k\end{array}\right]$ the set of $k$-dimensional subspaces of $W$, when $W$ is a vector space. The geometric design $\mathrm{PG}_{e}(2 e, q)$ has $[V]$ as the set of points, and $\left\{[W] \mid W \in\left[\begin{array}{c}V \\ e+1\end{array}\right]\right\}$ as the set of blocks. The block graph of this design, where two blocks $\left[W_{1}\right],\left[W_{2}\right]$ are adjacent whenever $\operatorname{dim} W_{1} \cap W_{2}=e$, is the Grassmann graph $J_{q}(2 e+1, e+1)$ which is isomorphic to the Grassmann graph $J_{q}(2 e+1, e)$.

For each prime power $q$ and an integer $e \geq 2$, the twisted Grassmann graph $\tilde{J}_{q}(2 e+1, e)$ discovered by Van Dam and Koolen is a distance-regular graph with the same parameters as the Grassmann graph $J_{q}(2 e+1, e)$. The twisted Grassmann graphs were the first family of non-vertex-transitive distance-regular graphs with unbounded diameter. We refer the reader to $[2,3]$ for an extensive discussion of distance-regular graphs, to [10] for a characterization of Grassmann graphs, and to $[1,5]$ for more information on the twisted Grassmann graphs.

Jungnickel and the second author [9] constructed a family of designs which have the same parameters as $\mathrm{PG}_{e}(2 e, q)$, and showed that these designs give 


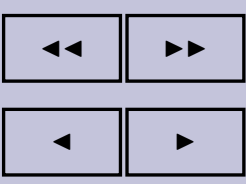

page $3 / 6$

go back

full screen

close

quit

Theorem 1. The twisted Grassmann graph $\tilde{J}_{q}(2 e+1, e)$ is isomorphic to the block graph of the design $\left([V], \mathcal{A}^{\prime} \cup \mathcal{B}^{\prime}\right)$, where two blocks are adjacent if and only if their intersection has size $\left(q^{e}-1\right) /(q-1)$.

Proof. We define a mapping $f: \mathcal{A} \cup \mathcal{B} \rightarrow \mathcal{A}^{\prime} \cup \mathcal{B}^{\prime}$ by

$$
f(W)= \begin{cases}{[\sigma(W \cap H) \cup(W \backslash H)]} & \text { if } W \in \mathcal{A}, \\ {[\sigma(W)]} & \text { if } W \in \mathcal{B} .\end{cases}
$$

It suffices to show

$$
W_{1} \sim W_{2} \Longleftrightarrow\left|f\left(W_{1}\right) \cap f\left(W_{2}\right)\right|=\frac{q^{e}-1}{q-1} .
$$

If $W_{1}, W_{2}$ are subspaces of $V$, then

$$
\begin{aligned}
\operatorname{dim} \sigma\left(W_{1} \cap H\right) \cap \sigma & \left(W_{2} \cap H\right) \\
= & \operatorname{dim} \sigma\left(W_{1} \cap H+W_{2} \cap H\right) \\
= & 2 e-\operatorname{dim} W_{1} \cap H-\operatorname{dim} W_{2} \cap H+\operatorname{dim} W_{1} \cap W_{2} \cap H \\
= & \begin{cases}\operatorname{dim} W_{1} \cap W_{2} & \text { if } W_{1}, W_{2} \in \mathcal{A}, W_{1} \cap W_{2} \subset H \\
\operatorname{dim} W_{1} \cap W_{2}-1 & \text { if } W_{1}, W_{2} \in \mathcal{A}, W_{1} \cap W_{2} \not \subset H \\
\operatorname{dim} W_{1} \cap W_{2}+1 & \text { if } W_{1} \in \mathcal{A}, W_{2} \in \mathcal{B}, \\
\operatorname{dim} W_{1} \cap W_{2}+2 & \text { if } W_{1}, W_{2} \in \mathcal{B}\end{cases}
\end{aligned}
$$

Thus, if $W_{1}, W_{2} \in \mathcal{A}$, then

$$
\begin{aligned}
& \left|f\left(W_{1}\right) \cap f\left(W_{2}\right)\right| \\
& \quad=\left|\left[\sigma\left(W_{1} \cap H\right) \cap \sigma\left(W_{2} \cap H\right)\right]\right|+\left|\left[W_{1} \cap W_{2} \backslash H\right]\right| \\
& \quad= \begin{cases}\frac{q^{\operatorname{dim} W_{1} \cap W_{2}}-1}{q-1} & \text { if } W_{1} \cap W_{2} \subset H, \\
\frac{q^{\operatorname{dim} W_{1} \cap W_{2}-1}-1}{q-1}+\frac{q^{\operatorname{dim} W_{1} \cap W_{2}}-q^{\operatorname{dim} W_{1} \cap W_{2}-1}}{q-1} & \text { otherwise }\end{cases} \\
& =\frac{q^{\operatorname{dim} W_{1} \cap W_{2}}-1}{q-1},
\end{aligned}
$$

and hence (1) holds.

Similarly, if $W_{1} \in \mathcal{A}, W_{2} \in \mathcal{B}$, then

$$
\left|f\left(W_{1}\right) \cap f\left(W_{2}\right)\right|=\frac{q^{\operatorname{dim} W_{1} \cap W_{2}+1}-1}{q-1},
$$




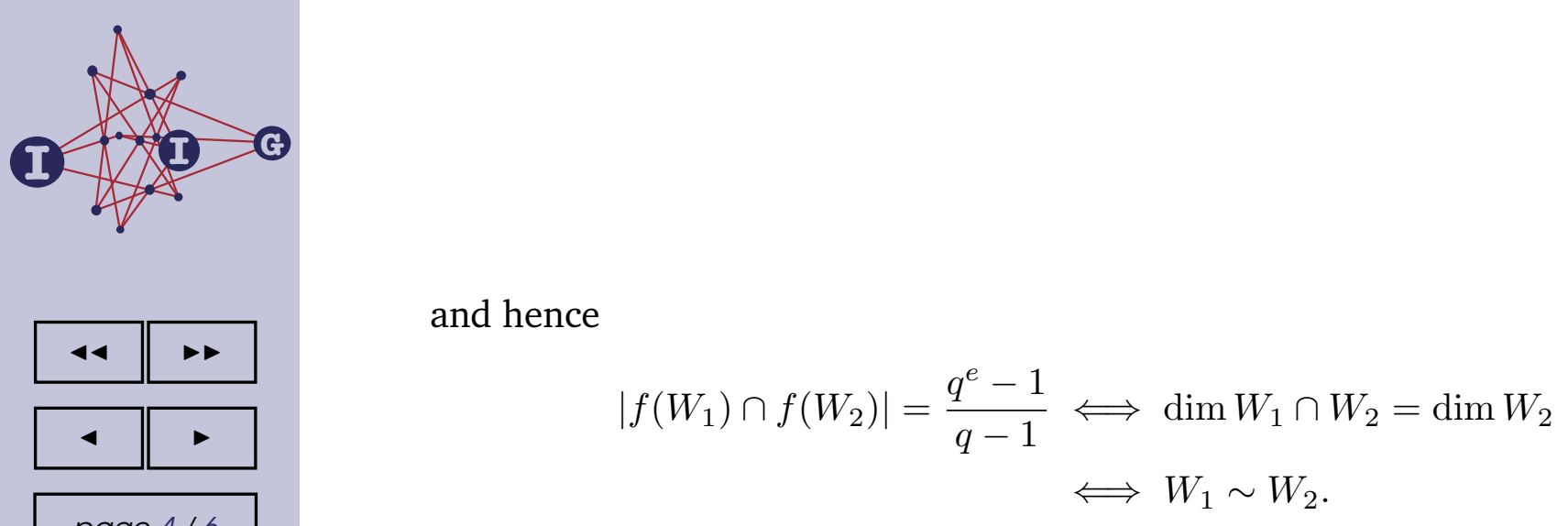

Finally, if $W_{1}, W_{2} \in \mathcal{B}$, then

$$
\left|f\left(W_{1}\right) \cap f\left(W_{2}\right)\right|=\frac{q^{\operatorname{dim} W_{1} \cap W_{2}+2}-1}{q-1} .
$$

and hence (1) holds.

\section{The automorphism group}

Let $\Gamma \mathrm{L}(V)_{H}$ denote the stabilizer of the hyperplane $H$ in the general semilinear group $\Gamma \mathrm{L}(V)$ on $V$. For each $\phi \in \Gamma \mathrm{L}(V)_{H}$, we define a permutation $\phi^{\prime}$ on $[V]$ by

$$
\phi^{\prime}(\langle x\rangle)= \begin{cases}\sigma \phi \sigma(\langle x\rangle) & \text { if }\langle x\rangle \in[H], \\ \phi(\langle x\rangle) & \text { otherwise, }\end{cases}
$$

where $\langle x\rangle$ denotes the 1-dimensional subspace spanned by a nonzero element $x \in V$. It is straightforward to verify that $\phi^{\prime}$ is an automorphism of the design $\left([V], \mathcal{A}^{\prime} \cup \mathcal{B}^{\prime}\right)$. Indeed, suppose $W \in\left[\begin{array}{c}V \\ e+1\end{array}\right], W \not \subset H$. Then

$$
\begin{aligned}
\phi^{\prime}([\sigma(W \cap H) & \cup(W \backslash H)]) \\
& =\left\{\phi^{\prime}(\langle x\rangle) \mid\langle x\rangle \in[\sigma(W \cap H) \cup(W \backslash H)]\right\} \\
& =\{\sigma \phi \sigma(\langle x\rangle) \mid\langle x\rangle \in[\sigma(W \cap H)]\} \cup\{\phi(\langle x\rangle) \mid\langle x\rangle \in[W \backslash H]\} \\
& =\{\langle x\rangle \mid \sigma \phi(W \cap H) \supset\langle x\rangle \in[H]\} \cup[\phi(W) \backslash H] \\
& =[\sigma(\phi(W) \cap H) \cup \phi(W) \backslash H)] \\
& \in \mathcal{A}^{\prime} .
\end{aligned}
$$

Next suppose $W \in\left[\begin{array}{c}H \\ e+1\end{array}\right]$. Then

$$
\begin{aligned}
\phi^{\prime}([W]) & =\{\sigma \phi \sigma(\langle x\rangle) \mid\langle x\rangle \in[W]\} \\
& =\{\langle x\rangle \mid \sigma \phi \sigma(W) \subset\langle x\rangle \in[H]\} \\
& =[\sigma \phi \sigma(W)] \\
& \in \mathcal{B}^{\prime} .
\end{aligned}
$$

Therefore, $\phi^{\prime}$ is an automorphism of the design $\left([V], \mathcal{A}^{\prime} \cup \mathcal{B}^{\prime}\right)$. 



\section{References}

[1] S. Bang, T. Fujisaki and J.H. Koolen, The spectra of the local graphs of the twisted Grassmann graphs, European J. Combin. 30 (2009), 638-654.

[2] E. Bannai and T. Ito, "Algebraic Combinatorics I", Benjamin/Cummings, Menlo Park, 1984.

[3] A.E. Brouwer, A.M. Cohen and A. Neumaier, "Distance-Regular Graphs", Springer-Verlag, Heidelberg, 1989.

[4] E.R. van Dam and J.H. Koolen, A new family of distance-regular graphs with unbounded diameter, Invent. Math. 162 (2005), 189-193.

[5] T. Fujisaki, J.H. Koolen and M. Tagami, Some properties of the twisted Grassmann graphs, Innov. Incidence Geom. 3 (2006), 81-87.

[6] N. Hamada, On the $p$-rank of the incidence matrix of a balanced or partially balanced incomplete block design and its application to error correcting codes, Hiroshima Math. J. 3 (1973), 154-226.

[7] N. Hamada and H. Ohmori, On the BIB-design having the minimum p-rank, J. Combin. Theory Ser. A 18 (1975), 131-140.

[8] J.W.P. Hirschfeld, "Projective Geometries over Finite Fields", 2nd edn., Oxford University Press, 1998.

[9] D. Jungnickel and V.D. Tonchev, Polarities, quasi-symmetric designs, and Hamada's conjecture, Des. Codes Cryptogr. 51 (2009), 131-140.

[10] K. Metsch, A characterization of Grassmann graphs, Europ. J. Combin. 16 (1995), 639-644.

Akihiro Munemasa

Graduate SCHOOL OF Information ScIENCES, TOHOKU UNIVERSITy, SENDAi, 980-8579 JAPAN

e-mail: munemasa@math.is.tohoku.ac.jp

Vladimir D. Tonchev

Department of Mathematical Sciences, Michigan Technological University, Houghton, MI 49931, USA

e-mail: tonchev@mtu.edu 